\title{
LITERATURE SURVEY ON EXISTING POWER SAVING ROUTING METHODS AND TECHNIQUES FOR INCREASING NETWORK LIFE TIME IN MANET
}

\author{
K. Mariyappan ${ }^{1}$ and M. Karnan ${ }^{2}$ \\ Department of Computer Science and Engineering, Aringer Anna College of Engineering and Technology, India
}

\begin{abstract}
Mobile ad hoc network (MANET) is a special type of wireless network in which a collection of wireless mobile devices (called also nodes) dynamically forming a temporary network without the need of any preexisting network infrastructure or centralized administration. Currently, Mobile ad hoc networks (MANETs) play a significant role in university campus, advertisement, emergency response, disaster recovery, military use in battle fields, disaster management scenarios, in sensor network, and so on. However, wireless network devices, especially in ad hoc networks, are typically battery-powered. Thus, energy efficiency is a critical issue for battery-powered mobile devices in ad hoc networks. This is due to the fact that failure of node or link allows re-routing and establishing a new path from source to destination which creates extra energy consumption of nodes and sparse network connectivity, leading to a more likelihood occurrences of network partition. Routing based on energy related parameters is one of the important solutions to extend the lifetime of the node and reduce energy consumption of the network. In this paper detail literature survey on existing energy efficient routing method are studied and compared for their performance under different condition. The result has shown that both the broadcast schemes and energy aware metrics have great potential in overcoming the broadcast storm problem associated with flooding. However, the performances of these approaches rely on either the appropriate selection of the broadcast decision parameter or an energy efficient path. In the earlier proposed broadcast methods, the forwarding probability is selected based on fixed probability or number of neighbors regardless of nodes battery capacity whereas in energy aware schemes energy inefficient node could be part of an established path. Therefore, in an attempt to remedy the paucity of research and to address the gaps identified in this area, a study examining the impact of residual energy on forwarding probability and how to exclude small amount of residual battery level during route establishment are critically important.
\end{abstract}

Keywords:

Mobile Adhoc Network (MANET), Mobile Hosts (MH), Adhoc On Demand Distance Vector (AODV)

\section{INTRODUCTION}

Mobile ad hoc network (MANET) [2], [3] is a self-organizing and self-configuring multihop wireless network, which is composed of a set of mobile hosts (MHs) that can move around freely and cooperate in relaying packets on behalf of one another. MANET supports robust and efficient operations by incorporating the routing functionality into MHs.

In Ad hoc Network the mobile nodes form a temporary network that communicates via short range wireless technologies such as Wireless-Fidelity (IEEE 802.11), Bluetooth (IEEE 802.15.1), ZigBee (IEEE 802.15.4) and Ultra-Wide Band (IEEE 802.15.3) without the aid of any preexisting infrastructure or centralized administration. The Fig. 1 shows the structure of a typical infrastructure-less networks.

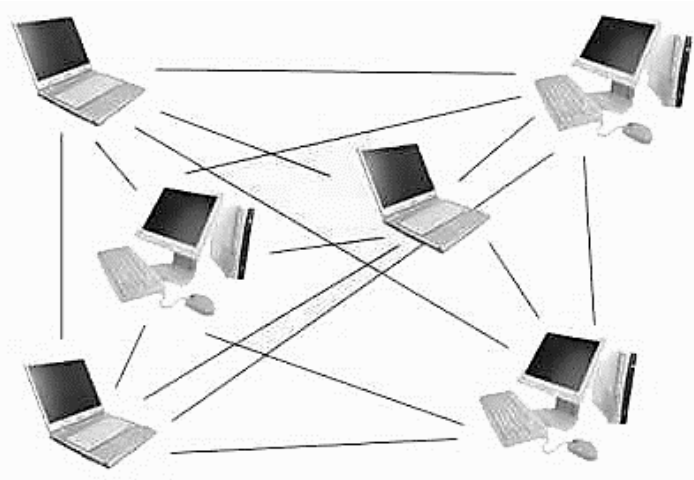

Fig.1. MANET

In MANETs, the unicast routing establishes a multihop forwarding path for two nodes beyond the direct wireless communication range. Routing protocols also maintain connectivity when links on these paths break due to effects such as node movement, battery drainage, radio propagation, and wireless interference

The power conservation techniques have been addressed in the literature by several scholars. This paper discus some of the existing power saving routing methods and techniques developed for mobile ad hoc networks. Management of energy resources in wireless ad hoc networks is of paramount importance for battery driven mobile nodes due to the limited availability of energy capacity. The methods have been developed to minimize the energy costs of communication since wireless communication consumes a considerable amount of energy or/and to find an energy efficient route for data communication by practicing energy aware routing strategies. The ultimate goals of such routing strategies are to increase the network lifetime, reduce energy consumption and/or distribute energy usage among mobile nodes.

The problem of energy efficiency in MANETs can be addressed at different layers of the protocol stack such as power saving medium access control layer protocols, energy efficient routing protocols, and power sensitive network architectures. However, this paper mainly discusses an energy efficient approaches which have been developed at the network layer.

The chapter is organized as follows. Section 2 discusses previous studies which minimize energy consumption of mobile node battery indirectly by reducing number of flooding packets and section 3 describes energy efficient techniques that minimize energy consumption and maximize network life time in ad hoc networks using energy aware metrics. Section 4 presents comparison of most of the aforementioned developed routing protocols and section 5 presents Conclusion and future work. 


\section{ENERGY EFFICIENT APPROACH USING BROADCAST SCHEMES}

Route discovery in mobile ad hoc networks is done by flooding. Flooding is the technique in which a node, receiving flooded packet $\mathrm{m}$ for the first time, retransmits it once [3]. In Classical Flooding [4, 5, 6], a source node broadcasts a message to all of its neighbors and each of these neighbors will examine whether the message has been seen before or not. If yes, the message will be dropped; otherwise the message will be rebroadcasted to their neighbors. The process will continue till every node on the network receives the message. Although flooding routing approaches are simple to implement, they result problems including redundant retransmissions, channel contentions, packet collisions, packet loss, and resource blindness (e.g. exhausts mobile node battery, bandwidth), leading them neither effective nor efficient for message dissemination in mobile ad hoc networks. Collectively the above mentioned problems are referred to as the broadcast storm problem $[4,5]$.

\subsection{PROBABILISTIC BASED METHODS}

In probabilistic approach, instead of retransmitting packets to all of its neighbors as a case in flooding, a node only rebroadcasts received packets with a predetermined forwarding probability of $P_{i}$ upon receiving it for the first time. In probabilistic approach, the choice of forwarding probability is an important parameter for its effectiveness. To select an appropriate forwarding probability, the authors in [7] have proposed the use of random graphs [8] and percolation theory [9] in MANETs. They claimed that there exists a probability value $P_{i}<1$, such that by using $P_{i}$ as a forwarding probability, almost all nodes receive a flooding packet, while a forwarding probability of $p$ greater than $P_{i}$ is not yield any significant improvement. Due to the non-existence of mathematical technique for estimating forwarding probability $P_{i}$ for any type of mobile ad hoc network, many probabilistic approaches use various predetermined value forwarding probability for $P_{i}$ to retransmit a newly received packets. The probabilistic schemes proposed in [10] aim at reducing routing packet redundancy. In this scheme, when a node receives a broadcast message for the first time, it rebroadcasts the message with fixed and pre-determined probability $p$ regardless of node's energy level and neighbor information. The researchers have shown that, the optimal value of $p$ in terms of high reach ability and saved rebroadcast is approximately equal to 0.65 to 0.7 . Their approaches reduce overheads but might suffer from frequent broken link and energy consumption due to negligence of the impact of node degree/number of neighbors and energy level of each node i.e. the assignment of the forwarding probability is fixed irrespective of each node conditions.

\section{Algorithm: Fixed probabilistic scheme (FP)}

On receiving a broadcast packet $m$ at a node $X$

If (packet $m$ is received for the first time)

Forward $m$ with a probability $p$

Else

Drop the packet $m$

End algorithm
The advantage of probabilistic broadcasting over the other proposed broadcast methods $[4,11,12]$ is its simplicity. Even if probabilistic broadcast methods can significantly reduce the impact of the broadcast storm problem, an early death of packet is a snag for the approaches $[4,5,7]$. However the authors in [13, 14] have argued that poor reach ability of packets occurred by the probabilistic broadcast algorithms in $[4,5,7]$ is due to the assignment of the same forwarding probability at every node in the network regardless of the number of its neighbors. Thus, to resolve the reachability problems, references in $[13,14]$ have proposed fixed pair of probabilistic broadcast scheme where the forwarding probability $p$ is adjusted based on node degree information. Node degree is obtained by periodical exchange of "HELLO" packets between neighbors. However, an adaptive probability based on number of neighbors regardless of node battery level exhausts node battery because the rebroadcasting probability is adjusted irrespective of node's residual energy which exposes low battery capacity nodes equally participate during path construction.

Another study that focused on an adaptive probability value based on number of neighbors was proposed by [15]. The author tried to overcome the drawbacks of fixed probability. On receiving the first flooding packet by a node, if the node degree of the node is smaller than node degree of the network then the node re-forwards the message with high probability otherwise it reforwards with smaller value of probability. The approach has two shortcomings. First, the scheme did not consider energy level of the intermediate nodes and hence low capacity of node's battery might be part of a route during route discovery process. The second one is the difficulty of obtaining the value of an average node degree of the network.

\subsection{COUNTER-BASED METHODS}

In a counter based method, when a mobile node receives a broadcast packet, it initiates a random assessment delay (RAD) timer and a counter which counts the number of received copies $C$ for each packet $[16,17,18]$. The RAD time is a randomly chosen time between 0 and $T_{\max }$ seconds in which a node should wait for additional packets and $T_{\max }$ is the maximum possible waiting delay for a node. This delay has the following advantage. First, it allows nodes to receive sufficient amount of redundant packets so that a node will decide to retransmit or not. Second the randomized selection of delay time reduces the probability of packet collision. When the RAD timer expires, the node reforwards the packet only if the counter is below a particular threshold value $C$. If the counter exceeds the threshold value after expiration of RAD timer, the node assumes that all its neighbors have received the same packet, and drops the flooding packet. The predetermined counter threshold $C$ is the key parameter in this scheme. The authors in $[4,5,11]$ have demonstrated that broadcast redundancy associated with simple flooding can be reduced by choosing a threshold value between 1 and 4 while maintaining comparable reachability in a network. The author in [17] is also recommended $0.1 \mathrm{sec}$ as an optimal time for RAD which minimizes end-to-end delay and rebroadcasted packets by achieving an acceptable reachability. However the approach did not have notion of nodes remaining battery level. 


\section{Algorithm: Counter Based Scheme(CB)}

Upon reception of a broadcast packet $m$ at a node $x$ for the first time

Initialize the packet counter $c$ to 1

Set and wait for RAD to expire

While waiting;

For every duplicate packet $m$ received

Increment $c$ by 1

If $(c<C)(C$ is the counter threshold $)$

Forward the packet $m$

Else

Drop the packet $m$

End algorithm

\subsection{AREA-BASED METHOD}

Area based method allows a mobile node to forward a broadcast message based on the additional coverage area which is determined by a distance-based scheme or location-based scheme. For example, if a node receiving the message is located a few meters far from the sender, the additional area covered by forwarding the message is quite small. On the contrary, if the mobile node receiving the message is located at the boundary of the sender's transmission range, then a retransmit would reach a significant additional area, 61\%, as suggested in [19]. We note that area based schemes only consider the coverage area of a transmission; they don't consider whether nodes exist or not within that area. The following is the discussion of the two ways distributing a message using area based scheme.

The first type of area based scheme is using the distance based scheme. A node using the distance based scheme requires that nodes know the distance between itself and each neighboring node that has previously rebroadcast a given packet. This could be handled by a GPS receiver, where each node adds their location information during packet transmission or by physical layer parameter such as the signal strength at a node from the received packet. Upon reception of the first unseen packet by node $\mathrm{B}$ from node $A$, the distance $\|A B\|$ is calculated. If the distance $\|A B\|$ is below the threshold distance value, the message is dropped otherwise a node initiates a random assessment delay timer (or RAD for short) and waits for redundant packets from any node $C$. When the RAD timer expires, the distance $\|B C\|$ for each node $C$ is examined to see if any of the sender nodes is less than the threshold distance value. If true, the node does not rebroadcast. Otherwise, the node retransmits it. This is because the authors assumed that only a small portion of the area can be covered when a nearby neighbor already transmitted the message. However the distance-based scheme suffers from redundant number of broadcast packets. This is due to the fact that every node that received a broadcast packet still rebroadcast the packet if all of the transmission distances are above threshold value. Furthermore the scheme did not have notion of energy.

\section{Algorithm: Distance Based Scheme (DB)}

Upon reception of a broadcast packet $m$ at a node B for the first time
If (the distance between the sender and node B is greater than the threshold distance $D$ )

Initiate a waiting timer (RAD)

Before the timer expires;

Check the distance of each sender of packet $m$

If (any of the distance between each sender and node $B$ is below the threshold distance $D$ )

The packet $m$ is dropped

Else

Forward the packet $m$ after the RAD expires

Else

The packet $m$ is dropped

End algorithm

The second type of Area based method is Location based Scheme, such as [20]. In location based scheme, each node must have the means to know its own location e.g. Global Positioning System (GPS). Whenever a mobile node originates or rebroadcasts a packet it adds its own location to the header of the packet it broadcasts. When a neighboring node initially receives the packet, it records the location of the sender and calculates the additional coverage area. If the additional area is less than a threshold value, the node will not rebroadcast, and all future receptions of the same packet will be ignored. Otherwise, the node initiates a RAD timer and waits for redundant packets. For each received redundant packets during the $\mathrm{RAD}$, the node recalculates the additional coverage area and updates the stored value of the additional covered area. The packet is dropped as soon as the recomputed additional coverage area falls below the threshold value otherwise the packet is forwarded after its timeout. The authors note that when multiple copies of packets are received from node's neighbors, the additional coverage approaches zero rapidly. The most challenging task for such scheme is the cost of calculating an additional coverage, which is related to calculating many intersections among several circles. This problem will be worse when a node has many neighbors. Furthermore the scheme has no notion of node battery capacity.

\section{Algorithm: Location Based Scheme(LB)}

Upon reception of a broadcast packet $m$ at a node $x$ for the first time

If (the coverage area is larger than the threshold Location $L$ )

Initiate a waiting timer (RAD)

Before the timer expires;

Re-compute the coverage area covered by received packet $m$

Else_If (any of the re-computed coverage area $<$ threshold location $L$ )

The packet $m$ is dropped

Else

Forward the packet $m$ after the RAD expires

Else

The packet $m$ is dropped

End the algorithm 


\subsection{GOSSIP BASED METHODS}

Gossip algorithms [21-24] were primarily intended to reduce the number of message flow within the network. Several variants of gossip-based protocols are proposed. Examples of the designed Gossip-based protocols are Push \& Pull, Information via Negotiation (SPIN) [23, 25], GOSSIP1 $(p, k), \operatorname{GOSSIP} 2(p 1, k, p 2, n)$ and $\operatorname{GOSSIP} 3(p, k, m)$ [21]. Each of the designed gossip based protocols utilizes local information in various ways. For example, GOSSIP1 $(p, k)$ retransmits each newly received messages with probability of 1 for the first $\mathrm{k}$ hops and with probability $p$ for the remaining number of hops. When the value of both parameters of $\operatorname{GOSSIP} 1(p, k)$ is 1 i.e. GOSSIP1 $(1,1)$, it is similar to flooding. In GOSSIP1 $(p, k)$, the parameter $k$ is used to minimize the likelihood of an early death of gossiping packets. One drawback of GOSSIP1 $(p, k)$ approach is an early death of gossiping packet. However to minimize shortcomings of $\operatorname{GOSSIP} 1(p, k), \operatorname{GOSSIP} 2$ $(p 1, k, p 2, n)$ was proposed. GOSSIP2 $(p 1, k, p 2, n)$ performs in a similar fashion to GOSSIP1 but it introduces two new variables $p 2$ and $n$. In GOSSIP2 ( $p 1, k, p 2, n)$ approach, if a node has fewer than $n$ neighbors, a node retransmits with probability $p 2$ rather than $p 1$ where $p 2>p 1$. Finally $\operatorname{GOSSIP} 3(p, k, m)$ approach is also an extension to $\operatorname{Gossip} 1(p, k)$ except if a node with $n$ neighbors receives a message and does not broadcast it, but then does not receive at least $m=p n$ duplicate messages from its neighbors within a gossiping timeout period, the packet is forwarded to all its neighbors; otherwise it is dropped. For instance, Haas et al. [22] showed that $\operatorname{GOSSIP3}(p, k, m)$ protocol reduces up to $35 \%$ messages than flooding in MANETs. In the networks they have considered gossiping probability between 0.6 and 0.8 and shown that with a gossip probability of 0.65 i.e. $\operatorname{GOSSIP} 3(0.65,1,1)$ almost every node gets the message in every execution. Furthermore, their simulation results showed that there is a bimodal effect with an average node degree of 8 . The scheme has good performance in reachability and reduction of message overhead as compared to other flooding. However the major drawback of the scheme is an assignment of fixed gossiping probability during the design time regardless of node energy and number of node neighbors.

As can been seen from this section the proposed broadcast routing schemes did not consider node's battery as a cost metric during route establishment. Hence, routing techniques which did not consider energy as a metric tend to use energy inefficient path during data communications which results frequent broken link and network partition leading to poor packet delivery ratio beyond consuming precious resources of node battery.

\section{ENERGY EFFICIENT APPROACH USING ENERGY AWARE SCHEMES}

The second category of energy efficiency of MANETs is based on energy aware routing approach. The main goal of energy-aware routing protocols is to minimize the energy consumed by mobile nodes, increasing the network lifetimes and/or distribute loads among mobile entities. In the recent past years energy efficient routing in Ad hoc network was proposed by many research works. The majority of energy efficient routing protocols for MANET tried to reduce energy consumption by means of an energy efficient routing metric instead of the minimum-hop metric. Each and every protocol has its own advantages and shortcomings. None of them can perform better in every condition. This section documents some of the many energy efficient schemes developed by researchers in the field along with their shortcomings.

The minimum total power routing (MTPR) proposed in [26, 27-37] uses the transmission power as the cost metric. The cost function is defined as follows:

$$
C_{R}=\sum_{i=1}^{k-1} P_{T}(i)
$$

where, $P_{T}(i)$ is the transmission power of node $i$ and $C_{R}$ is the total transmission power for route $R$. Then, the MTPR scheme selects the route having the minimum cost value i.e. the minimum summation of transmission power among each route between source and destination, $P_{M T P R}$

In MTPR, the optimal route is found using:

$$
P_{\text {MTPR }}=\min _{R \in S} C_{R}
$$

where, $S$ is the set containing all possible routes. Consider Fig.2, where the number above, inside and below the circle represents a cost metric, node address and residual energy of each node respectively. In the Fig.2, the cost of each node is the amount of transmission power required per bit. For instance node 1 requires .02 joule to transmit a bit to its neighbors. Hence the cost of path S-1-3-5-7-D and path S-2-4-6-8-D is 0.09 and 0.11 joule respectively. Since MTPR selects a path having minimum summation of transmission power per bit, path S-1-3-5-7-D will be selected for data packet transmission. However, this path consists of node's having very low residual energy which results frequent broken link, leading to network lifetime reduction. Note that if all the wireless devices have the same transmission power; the MTPR will be the same as the minimum hop count routing protocol

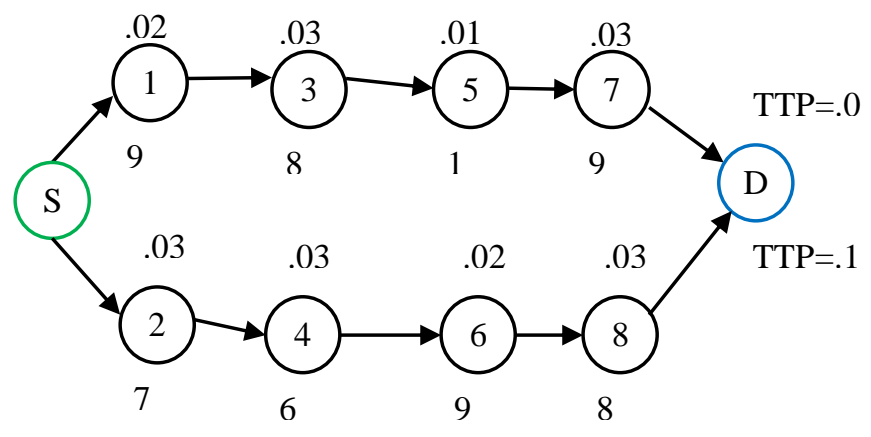

Fig.2. MTPR route establishment

In [37], the authors proposed the Minimum Battery Cost Routing (MBCR) based on the reciprocal of remaining energy capacity as a cost metric. The idea of this metric is directly related to an increase in reluctance of the node to participate in routing when the residual battery power decreases and the cost metric function is defined as follows:

$$
C_{R}=\sum_{i=1}^{k-1} f\left(E_{r}^{i}(t)\right)
$$

where, $f\left(E_{r}^{i}(t)\right)=\frac{1}{E_{r}^{i}(t)}$ and $E_{r}^{i}(t)$ is the remaining energy of node $i$ at time $t$. 
MBCR selects routes with a minimum cost value, $P_{M B C R}$, to ensure the route with the maximum remaining energy capacity will be selected.

$$
P_{M B P R}=\min _{R \in S} C_{R}
$$

where, $S$ is the set containing all possible routes.

For instance in Fig.3, the cost of node 1 is .01 which is the reciprocal of the residual energy of node 1 . Hence, the Total Battery Cost (TBC) of route S-1-3-5-7-9-D and route S-2-4- 6-8$\mathrm{D}$ is 0.7 and 0.72 respectively. Therefore, MBCR algorithm selects route S-1-3-5-7-9-D for data transmission as it has the smallest cost metric among the possible routes. However, the selected path for data transmission will be quickly broken after a few data packets sent through it due to the inclusion of smaller residual energy node i.e. node 5. Since MBCR only considers the summation of the reciprocal of remaining energy capacity, little energy capacity node can still be included by the selected route. Hence some nodes could run out of battery leading to sparse network connectivity and network partition. Furthermore, Flooding is used to find a route between source and destination which results packet collision, channel contentions and battery exhaustion

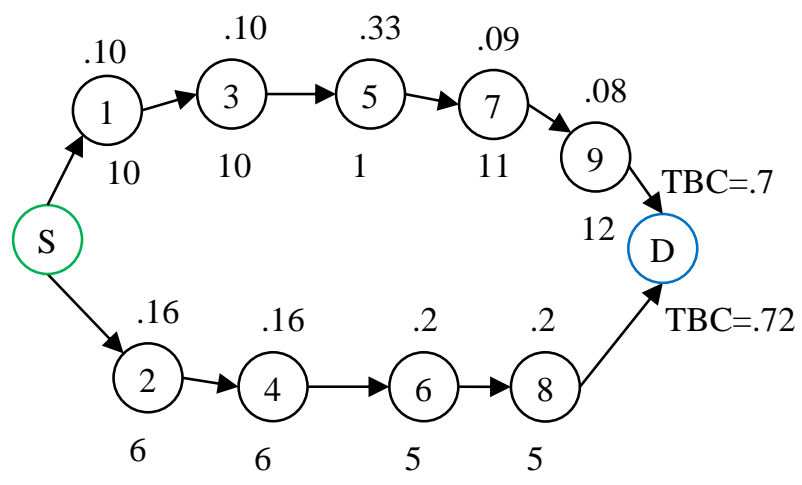

Fig.3. MBCR route establishment

In [38], Jin-Man Kim and Jong-Wook Jang proposed an enhanced AODV routing protocol to maximize networks lifetime in MANET using an Energy Mean Value algorithms. Here, energy remaining of each node in the path between source and destination is accumulated and delivered to the destination by adding a field on a RREQ message. The destination node does not give a RREP reply immediately for the first RREQ, rather it waits for $3 \times$ NODE_TRAVERSAL_TIME to receive duplicate RREQ packets destined for the node. Then the destination node adds the accumulated residual energy of each path and divides by the number of hops along the paths to obtain the mean energy of network. Finally the destination node adds mean energy on RREP packets and unicasts it along the reverse path of the RREQ message received first.

Then, nodes hearing the RREP message store the mean energy on their routing table. When a new path is explored, the mean energy stored in each node is compared with the energy remaining in the node. If the remaining energy is less than the mean energy, the delay time of RREQ message is set to be $0.5 \mathrm{~ms}$ otherwise the delay time of RREQ message is set to $0.05 \mathrm{~ms}$. The drawback of the scheme is twofold; first, flooding is used as a route discovery strategy. Second mean energy, which is difficult to obtain an average of the residual energy of all nodes on the network, is used as a cost metric instead of residual energy of each node which decreases the lifetime of the network.

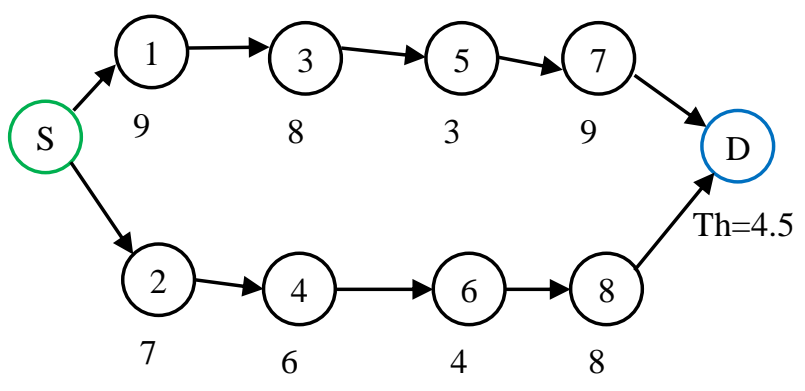

Fig.4. Local Energy Aware Route Establishment

In [39-42], proposed Local Energy-Aware Routing. When a node received a RREQ message at time $t$, it compared its current remaining energy capacity with the predefined threshold value or computed value. If the residual energy is less than the threshold or computed value, the RREQ message is dropped. Otherwise, the message is processed and forwarded. However in this scheme, the destination will receive a route request message when all intermediate nodes along the route have enough battery levels. If all the paths to destination node have less residual energy than the threshold, the RREQ message will not be reached at the destination. For example in Fig.4, let us assume that the energy threshold $(\mathrm{TH})$ value of the scenario is 4.5 joule. From the scenario, even if node $S$ initiates a RREQ packet to find destination node $D$, the packet will not reach to destination node $D$ because there are intermediate nodes along the path (i.e. node 5 and node 6) whose residual energies are below the threshold value

The work done in [43] proposed Alternate Link Maximum Energy Level (ALMEL-AODV) which considers node remaining energy as a routing metric to balance and extend the life time of the nodes in the network. The proposed algorithm adds a field, which keeps the sum of residual energy of the route, on RREQ packets. The destination node chooses two highest summations of residual energy paths for data transmission and sends back to the source node. The second path will be used as a backup if the first path fails.

Although the metric used is important, a node which has very low residual energy might be selected for data transmission as they consider the maximum summation of remaining energy. Hence the remaining capacity of each host should be consider as a metric beyond summation of residual energy to prolong the life time of the network and distributes loads among the mobile nodes. Furthermore, flooding is used as a route discovery mechanism, leading to exhaustion of each mobile node battery. Finally, the second path, which will be used later for data transmission if the first one fails, could not be an active path at the required moment due to the mobility of nodes in MANETs.

In [44], to mitigate the energy saving problem, an energyaware routing for Ad Hoc networks is proposed. The algorithm selects routing according to the dynamic priority-weight $(\beta)$ and takes the hop count as optimization condition. The dynamic priority weight is determined using the square of the ratio of residual battery energy $(R)$ and consumed energy $(C)$ of a node at time $t$ as shown, 


$$
\beta_{i}(t)=\left[\frac{R_{i}(t)}{C_{i}(t)}\right]^{2}
$$

The destination node selects two maximum summation of priority-weight which spends less energy and owns larger capacity based on synthetic analysis among possible routes and propagates the route reply (RREP) messages to the source node. The second path will be used when the primary path fails. Since the work considered the summation of priority-weight without a threshold, the selected path for data transmission might contain a node which has less remaining energy. Moreover the second path, which will be used later for data transmission if the first one fails, could not be an active path at the required moment due to the mobility of nodes in MANETs.

The authors in [45] proposed a PS-AODV routing protocol based on load conditions of a node to balance uneven nodes energy consumption of the traditional AODV. The authors made an improvement during route discovery process. Node checks its load value when it receives an RREQ packet before retransmission. If the load is too high, it refuses to forward the RREQ packet until the load is reduced. The metric value of load balance at the $i^{\text {th }}$ node is calculated as follows:

$m_{i}=b_{i} \times l_{i} ; b_{i}$ is the percentage of usable energy of node and $l_{i}$ is the length of the waiting or used queue. Based on the product of usable energy and load value of node, each node in the network has three states as follow: Paralysis: $m>=a$; Congestion: $b<=m$ $<=a$; Normal: $m<b$; where $a, b$ are a predetermined congestion factor.

Using the above metric each intermediate node decides whether a received RREQ packet is forwarded or not. When a node is in a "paralyzed" condition, it will drop all received RREQ. When a node is in "congestion" condition, it will reply an RREP only if there is an existing route in the routing table otherwise the RREQ packets will be dropped. When a node is in "normal" condition, it compares the value $\mathrm{m}$ in the RREQ and the metric value of the node itself. Then, the maximum value of $m$ is recorded on the routing table before forwarding. For duplicate RREQ packets, a node will compare the value of $m$ on the RREQ with $m t$ in the routing table. If the value of $\mathrm{m}$ is smaller than the value of $m t$ on the routing table, a node drops the RREQ packet otherwise it will update the value of $m t$ in the routing table and sent node pointer back to the previous hop. Furthermore to minimize congestion, RREQ is not forwarded. When a destination node receives the first RREQ, it waits for some time to obtain more routes, then choose the route having the minimum value of $\mathrm{m}$ and reply a RREP packet back to the source node. Since the technique considers the product of queue and energy as a cost metric, there is a high probability of choosing a node having less energy with smaller queue load. Hence the product metric could not give guarantee to protect nodes with little battery capacity which affects network life time. Furthermore if the intermediate nodes are in a paralyzed or congestion state no routing packet reaches to destination node. The update mechanism of the approach during normal state for duplicate packets is also not effective as congested node on the route replaces the node on the route having relatively less congested.

The paper in [46] proposed an energy saving AODV called AODVI. AODVI integrates residual energy and hop count as a cost function. They introduce a total power of the link $(P)$ field in
RREQ, RREP and routing table which keeps an updated routing cost value. The proposed algorithm categorizes each node in to $N$ levels by residual energy. The routing cost function of node $j$ at time $\mathrm{t}$ is calculated as: Metric $j=\alpha \min \left(L_{i}\right)+1 / H_{j} ; \alpha$ is a coefficient ( $0<=\alpha<=1), L_{i}$ is the energy level of node $i$ at time $t$, $H_{j}$ is the hops of path $j$. Then the destination node selects an optimal routing price function as follows:

Metric $=\max \{$ Metric $j \mid j \in G\} ; G$ is the set of possible routes

When each intermediate node receives an RREQ packet, first it determines its own cost value and compare with the cost value on the RREQ. The cost value of the RREQ packet is updated by the minimum cost value before retransmitted to its neighbors. Moreover each intermediate node keeps an updated cost value on the routing table for repeated RREQ packets. When receiving the same RREQ packet, the node compares the Metric and sequence number with the information stored in the routing table. If receiving larger sequence number, or the same sequence number but larger routing cost value, the node will update reverse routing information, and then retransmit the RREQ again to its neighbors, otherwise discard the RREQ. However the algorithm did not consider fair distribution of energy usage across nodes on the network. Furthermore flooding method is used for route discovery process and a node could retransmit the same packets several times leading to more number of routing overhead.

The authors [47] introduce an algorithm which combines Transmission Power and Remaining Energy Capacity and integrates these metrics into AODV so that the Ad hoc network has a better life time and the energy consumption across the nodes is reduced. During route discovery process the transmission and remaining energy values along the route are kept in the RREQ packets. At the destination or intermediate node these values are copied into the RREP packet and then transmitted back to the source node. The source node uses the maximum remaining energy capacity route and minimum transmission route alternately every time it performs route discovery. Since the metric did not consider hop count during selection of optimal route and transmission power is used for route selection, the selected link for data transmission could be frequently broken which causes more energy consumption and small network lifetime. Furthermore flooding is used as a route discovery technique between source and destination.

Kumar and Banu [48] present an energy Efficient Ad hoc on Demand Vector (E2AODV) scheme to balance load distribution of nodes. A threshold value is used to judge if intermediate node was overloaded or not. Here, an intermediate node receiving the RREQ will compare its current queue length with its threshold before rebroadcasting it. If queue length is greater than the threshold, the RREQ will be dropped. Otherwise, the node will broadcast it. In their scheme, the threshold value plays the key role in selecting nodes whether or not to forward RREQ. Every time an intermediate node receives a RREQ, it will recalculate the threshold according to the average queue length of all the nodes along the path to the node itself. Therefore, the threshold is variable and changing adaptively with the current load status of network. However the scheme did not consider residual energy as a cost metric which causes frequent broken link and retransmission of routing packet leading to energy consumption.

The authors in [49] proposed an energy based AODV (EBAODV) protocol. In EBAODV, when a source node transmits 
a route request packet it specify the amount of energy that each intermediate node should have during the transmission of data packets. When the intermediate node receives a RREQ packet, first it checks if it has enough available residual energy for the request. A node which does not satisfy the energy constraint will avoid retransmitting of RREQ packet. The approach entirely related to the amount of energy required by the source node to transmit its data packet. Therefore the path which consists of small amount of node battery level could be selected. For instance, if the source node requires a small amount of energy to transmit its data packets, the minimum residual energy node might be part of the route for data transmission which minimizes network lifetime leading to frequent broken link and node failure. The route discovery process could be flooding if the source node assigns small amount of residual energy as most of intermediate nodes' residual energy is expected above it. Furthermore the source node should know the amount of transmitting data ahead.

The approach proposed in [50] referred as EA-AODV classified nodes into the following three energy zones based on their remaining energy capacity:

- Normal Zone: The remaining energy capacity of a node is above $20 \%$ of its initial value.

- Warning Zone: The remaining energy capacity of a node is between $10 \%$ and $20 \%$ of its initial value.

- Danger Zone: The remaining energy capacity of a node is below $10 \%$ of its initial value.

The cost function is defined as,

$$
C_{R}=\sum_{i=1}^{k-1} C(i)
$$

$C(i)$ represents the cost of node $v_{i}$ and $C(i)$ could be $C_{\text {normal }}$, $C_{\text {warning }}$ or $C_{\text {danger }}$, which is calculated based on the energy zones defined above. $C_{\text {normal }}, C_{\text {warning }}$ or $C_{\text {danger }}$ are predefined values such that $C_{\text {normal }}<C_{\text {warning }}<C_{\text {danger }}$. In addition to the residual energy, the authors also consider number of neighbors during calculating cost function when a node is either in warning or danger state. The cost function of node $v_{i}$ is calculated as: $C(i)=$ $C_{\text {normal }}, k_{i} C_{\text {warning }}$ or $k_{i} C_{\text {danger }}$ : where $k_{i}$ is proportional to the number of neighbors of node $v_{i}$, i.e. the number of neighbors divided by five.

The destination node selects a route having minimum value of $C_{R}$ from the possible routes. In the scheme, even though a node is in the Warning or Danger Zone, it can still be chosen on a route due to the summation cost metric used by the scheme. Hence frequent link failure might be occurred due to the selection of energy incapable nodes, leading to reduction of network lifetime and depletion of node energy. Furthermore flooding is used to discover route between source and destination during route discovery process.

In the work proposed by [51], they presented a scheme which considers power during route discovery. The scheme deals with mobile ad hoc network having large number of nodes and handles a different data traffic levels. Their scheme modified AODV protocol by assuming that battery status has three states as shown below:

- If (battery status $<20 \%$ ), then it is in danger state.
- If $(20 \%<$ battery status $<50 \%)$, then it is in critical state and,

- If (battery status $>50 \%$ ) it is in active state.

The proposal by [51] indicate that only the node in active state are participating during route discovery and the rest nodes go to in a sleep state. However when the radio interface of a destination node is switched off, establishing a route to destination node is impossible which makes a source node reinitiate the route discovery process. Furthermore intermediate nodes could be in a sleep mode so that the intended destination node could be unreachable. Consequently a number of re-initiation of route discovery process could be occurred leading to a considerable amount of node's battery consumption.

The authors of PEER protocol in [52] introduce a route discovery technique along with progressive route maintenance procedure. PEER finds a set of shortest hop paths available and chooses the one having lowest energy consumption. During route discovery PEER first searches a path near the most energy efficient route between the source and the destination quickly, and then adjusts the nodes progressively so that the route would be energy efficient every time. A link cost table is used at each node to discover a more energy efficient route. Since the protocol did not consider residual energy during route discovery, low residual energy node could be part of the route which causes reduction of network lifetime and more number of retransmission of packets.

The Location Aided Routing (LAR) proposed by [53] assumes that each node knows its location through a GPS. LAR improves the efficiency of the route discovery procedure by limiting the scope of route request flooding by using location information. In LAR, a source node estimates the current location range of the destination based on information of the last reported location and mobility pattern of the destination. In LAR, an expected zone is defined as a region that is expected to hold the current location of the destination node. During route discovery procedure, the route request flooding is limited to a request zone, which contains the expected zone and location of the sender node.

In [53], two different schemes are used. In the first scheme, the source node calculates the expected zone which defines a boundary where the route request can travel to reach the destination in the request packets, and then starts a route discovery. Receiving the route request, a node forwards the request if it falls inside the request zone; otherwise it discards the request. When the destination receives the request, it replies with a route reply that contains its current location, time and average speed. The size of a request zone can be adjusted according to the mobility pattern of the destination. When speed of the destination is low, the request zone is small; and when it moves fast, the request zone is large.

In second scheme, a source node stores the coordinates of the destination in the route request packets and the relative distance among them. When a node receives a route request, it calculates its distance to the destination node and forwards the request only if the relative distance to the destination becomes smaller than the distance on the request packet; otherwise it discards the route request. Before forwarding the request, each node replaces the relative distance on the route request packet by its own distance. Both schemes limit the control overhead transmitted through the network and hence conserve energy and bandwidth. The disadvantage of LAR is that each node is required to have a GPS 
and obtaining precise location information may be difficult in some environments (for instance, GPS does not work well indoors, and proximity does not guarantee connectivity). Another drawback is (especially for the first method), that the protocol may conduct similar to flooding protocols in highly mobile networks.

The Energy Efficient Location Aided Routing (EELAR) Protocol in [73] was proposed based on the Location Aided Routing (LAR) [53] to reduce the energy consumption of the mobile node power. The protocol partitions the network area into six sectors with an assumption of a circular space centered at a reference node (base station). It uses location information to limit the flooding of routing packets during route discovery process on a sub-area (sector) containing the destination node instead of flooding throughout the network. Base station is used to maintain locations of mobile nodes in a position table. Despite the reduction of control packet overhead, feasibility of the protocol in mobile scenarios is doubtful. Furthermore, the need to update position table requires high energy consumption overheads. Moreover, each node requires additional hardware like GPS for the successful operation of the algorithm.

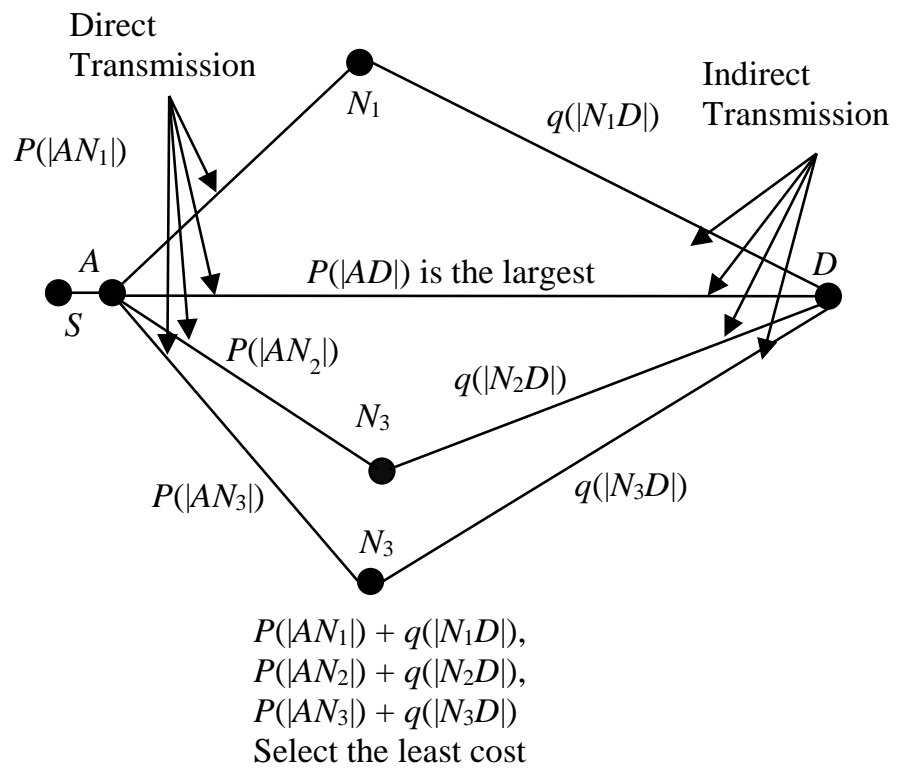

Fig.5. PLR route establishment

The Power-aware Localized Routing (PLR) protocol [54] implements a localized, fully distributed energy aware routing algorithm by avoiding global view information such as power level information of all nodes. However PLR assumes that a source node has the location information about the destination node and its neighbors. It is same as having the link costs from itself to the destination node and to its neighbors as well. Based on this information, the source node chooses the next hop through which the overall transmission power to the destination node is minimized but cannot find the optimal route. In data communication, a direct communication (i.e. from source to destination) may require more energy than an indirect communication (i.e. through intermediate nodes) due to the association between transmission energy and distance. In Fig.5, when node $A$ has a packet to send to node $D$, it can either send it directly to $D$ or through one of its neighbors $\left(N_{1}, N_{2}\right.$, or $\left.N_{3}\right)$. Note that $A$ to $N_{i}$ is a direct transmission while $N i$ to $D$ is an indirect transmission with some number of intermediate nodes between $N_{i}$ and $D$.

Therefore to choose the optimal route, node $A$ evaluates and compares the power consumption of each route candidate and selects one of its neighbors $\left(N_{1}, N_{2}\right.$, or $\left.N_{3}\right)$ as the next hop node which minimizes $p(|A N i|)+q(|N i D|)$ where $p$ and $q$ are the respective route costs. Disadvantage of PLR protocol is that a route which has less amount of remaining energy might be included during route establishment which results frequent retransmission of packets. Moreover, since hop count is not considered during route discovery, latency becomes another drawback.

The authors of $[55,56,57]$ propose a sleep/power-down mode technique. The main focus in sleep/power down approach is to minimize energy consumption during inactive time of communication. The authors argue that since most radio hardware supports a number of low power states, it is important to put the radio subsystem into the sleep state or simply turn it off to save energy. To reduce an early death of packet before reaching to destination during route discovery when some mobile nodes are in a sleep state, the papers propose an efficient node called a master node. The master node coordinates the communication on behalf of its neighboring slave nodes. Thus slave nodes, which is neighbor of master node, can safely sleep most of time and hence reduces battery energy consumptions. Each slave node periodically wakes up and communicates with the master node to check whether it has data to receive or not and go to sleep mode again if it is not addressed.

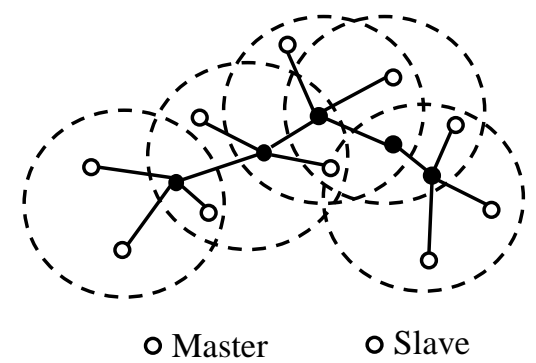

(a) Symmetric Power Model

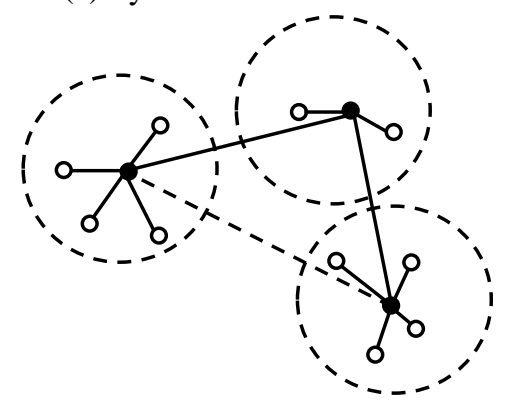

(b) Asymmetric Power Model

Fig.6. Master-Slave MANET architecture

In a mobile ad hoc network, more than one master node would be required because a single master cannot cover the entire network. The Fig.6 shows the master-slave network architecture, where mobile nodes except master nodes can save energy by putting their radio hardware into low power mode. The masterslave architecture in Fig.6(a) is based on symmetric power model, where master nodes have the same radio power and thus the same 
transmission range as slave nodes. On the other hand, Fig.6(b) shows the asymmetric power model, where master nodes have longer transmission range. Though master-slave type of network architecture has been widely studied for different reasons, such as interference reduction and ease of location management [29], the problem of selecting master nodes and maintaining the masterslave architecture under dynamic node configurations is still a challenging issue. Furthermore managing packets destined to slave nodes by a single master node is another big challenge as each mobile nodes of MANET are less capability in terms of memory, energy and processing. Examples of master-slave architecture are SPAN protocol [24] and Geographic Adaptive Fidelity (GAF) protocol [5] and put slave nodes in low power states to save energy Whereas, Prototype Embedded Network (PEN) protocol [55] employs the sleep period operation in an asynchronous way without involving master nodes.

Table.1. Comparison of Aforementioned Routing Protocols

\begin{tabular}{|c|c|}
\hline \multicolumn{2}{|c|}{ Protocol: AODV } \\
\hline Routing overhead & Very high \\
\hline Route selection metric & Hop count \\
\hline Special equipment & No \\
\hline objectives & Minimize hop count \\
\hline Network condition & Sparse \\
\hline \multicolumn{2}{|c|}{ Protocol: ALMEL-AODV } \\
\hline Routing overhead & High \\
\hline Route selection metric & $\begin{array}{c}\text { Maximum summation of residual } \\
\text { energy }\end{array}$ \\
\hline Special equipment & No \\
\hline objectives & Maximize network life time \\
\hline Network condition & Sparse \\
\hline \multicolumn{2}{|c|}{ Protocol: EBAODV } \\
\hline Routing overhead & High \\
\hline Route selection metric & Shortest path \\
\hline Special equipment & No \\
\hline objectives & Maximize network life time \\
\hline Network condition & Sparse \\
\hline \multicolumn{2}{|c|}{ Protocol: E2AODV } \\
\hline Routing overhead & High \\
\hline Route selection metric & $\begin{array}{c}\text { Minimum reciprocal of residual } \\
\text { energy }\end{array}$ \\
\hline Special equipment & No \\
\hline objectives & Maximize network life time \\
\hline Network condition & Sparse \\
\hline \multicolumn{2}{|c|}{ Protocol: PS-AODV } \\
\hline Routing overhead & High \\
\hline Route selection metric & Shortest path \\
\hline Special equipment & No \\
\hline objectives & Maximize network life time \\
\hline
\end{tabular}

\begin{tabular}{|c|c|}
\hline Network condition & Sparse \\
\hline \multicolumn{2}{|c|}{ Protocol: MTPR } \\
\hline Routing overhead & High \\
\hline Route selection metric & Transmission path \\
\hline Special equipment & No \\
\hline objectives & Maximum network life time \\
\hline Network condition & Sparse \\
\hline \multicolumn{2}{|c|}{ Protocol: local energy aware } \\
\hline Routing overhead & High \\
\hline Route selection metric & $\begin{array}{c}\text { Minimum reciprocal of residua } \\
\text { energy }\end{array}$ \\
\hline Special equipment & No \\
\hline objectives & Minimize routing overhead \\
\hline Network condition & Sparse \\
\hline \multicolumn{2}{|c|}{ Protocol: AODVI } \\
\hline Routing overhead & High \\
\hline Route selection metric & Transmission power \\
\hline Special equipment & No \\
\hline objectives & Maximize network life time \\
\hline Network condition & Sparse \\
\hline \multicolumn{2}{|c|}{ Protocol: LAR, EELAR, PLR } \\
\hline Routing overhead & Medium \\
\hline Route selection metric & Shortest path \\
\hline Special equipment & yes \\
\hline objectives & Minimize routing overhead \\
\hline Network condition & Dense \\
\hline \multicolumn{2}{|c|}{$\begin{array}{l}\text { Protocol: Sleep power down approach PEN, SPAN, } \\
\text { GAF }\end{array}$} \\
\hline Routing overhead & Medium \\
\hline Route selection metric & Shortest path \\
\hline Special equipment & yes \\
\hline objectives & Minimize routing overhead \\
\hline Network condition & Dense \\
\hline \multicolumn{2}{|c|}{ Protocol: Probability based scheme } \\
\hline Routing overhead & Low \\
\hline Route selection metric & Shortest path \\
\hline Special equipment & No \\
\hline objectives & Minimize routing overhead \\
\hline Network condition & Dense \\
\hline \multicolumn{2}{|c|}{ Protocol: Counter based scheme } \\
\hline Routing overhead & Low \\
\hline Route selection metric & Shortest path \\
\hline Special equipment & No \\
\hline objectives & Minimize routing overhead \\
\hline
\end{tabular}




\begin{tabular}{|c|c|}
\hline Network condition & Dense \\
\hline \multicolumn{2}{|c|}{ Protocol: Gossip based scheme } \\
\hline Routing overhead & Low \\
\hline Route selection metric & Shortest path \\
\hline Special equipment & No \\
\hline objectives & Minimize routing overhead \\
\hline Network condition & Dense \\
\hline \multicolumn{2}{|c|}{ Protocol: Distance based scheme } \\
\hline Routing overhead & Low \\
\hline Route selection metric & Shortest path \\
\hline Special equipment & Yes \\
\hline objectives & Minimize routing overhead \\
\hline Network condition & Dense \\
\hline \multicolumn{2}{|c|}{ Protocol: Location based scheme } \\
\hline Routing overhead & Low \\
\hline Route selection metric & Shortest path \\
\hline Special equipment & Yes \\
\hline objectives & Minimize routing overhead \\
\hline Network condition & Dense \\
\hline
\end{tabular}

Table.2. Comparison of Energy Aware Proposed Schemes using Energy Aware Metrics

\begin{tabular}{|c|c|c|c|}
\hline Protocol & $\begin{array}{l}\text { Number } \\
\text { of nodes }\end{array}$ & $\begin{array}{l}\text { Network } \\
\text { coverage } \\
\text { distance }\end{array}$ & $\begin{array}{c}\text { Delivery ratio } \\
(\%)\end{array}$ \\
\hline ALMEL-AODV & 50 & $500 \times 500$ & $95.1 \%$ \\
\hline EBAODV & 50 & $500 \times 500$ & $80 \%$ \\
\hline E2AODV & 50 & $800 \times 800$ & $\begin{array}{l}\text { Almost similar } \\
\text { to AODV }\end{array}$ \\
\hline PS-AODV & 50 & $500 \times 500$ & $85 \%$ \\
\hline AODVI & 50 & $500 \times 500$ & $92 \%$ \\
\hline EA-AODV & 50 & $1500 \times 500$ & $87 \%$ \\
\hline LAR,EELAR,PLR & 50 & \multirow{2}{*}{\multicolumn{2}{|c|}{$\begin{array}{l}\text { Require additional } \\
\text { equipment for location } \\
\text { information }\end{array}$}} \\
\hline PEN,SPAN,GAF & 50 & & \\
\hline
\end{tabular}

Table.3. Comparisons of Energy Efficient Techniques using Broadcast Schemes based on AODV

\begin{tabular}{|c|c|c|c|}
\hline Protocol & $\begin{array}{c}\text { Number } \\
\text { of nodes }\end{array}$ & $\begin{array}{c}\text { Simulation } \\
\text { area }\end{array}$ & $\begin{array}{c}\text { Average reduction of } \\
\text { routing overhead (\%) }\end{array}$ \\
\hline $\begin{array}{c}\text { Probabilistic } \\
\text { scheme }\end{array}$ & 10 to 50 & $800 \times 800$ & $30 \%$ \\
\hline $\begin{array}{c}\text { Counter } \\
\text { based scheme }\end{array}$ & 20 to 50 & $600 \times 600$ & $25 \%$ \\
\hline $\begin{array}{c}\text { Gossip based } \\
\text { scheme }\end{array}$ & 150 & $3300 \times 600$ & $35 \%$ \\
\hline
\end{tabular}

\begin{tabular}{|c|c|}
\hline \begin{tabular}{|c|} 
Distance \\
based scheme \\
\end{tabular} & Require additional equipment for location \\
\hline \begin{tabular}{|c|} 
Location \\
based scheme
\end{tabular} & information \\
\hline
\end{tabular}

\section{CONCLUSION AND FUTURE WORK}

In MANETs, flooding strategy is used to find a route between source and destination. Flooding route discovery process consumes enormous amounts of network resources due to the propagation of RREQ packets into the network, the so called the broadcast storm problem. Furthermore, MANET devices are energy constrained nodes. Hence the number of control messages transferred and received in the network dramatically affects the nodes 'power availability and consequently the existence of the network. This chapter investigated related research works that have been developed to reduce energy consumption of MANETs at the network layer Generally we categorized the proposed energy efficiency mechanisms at the routing level in to either of the broadcast schemes or energy aware schemes. We investigated both the broadcast schemes and energy aware metrics and the later have great potential in overcoming the broadcast storm problem associated with flooding. The result has shown that both the broadcast schemes and energy aware metrics have great potential in overcoming the broadcast storm problem associated with flooding. However, the performances of these approaches rely on either the appropriate selection of the broadcast decision parameter or an energy efficient path. In the earlier proposed broadcast methods, the forwarding probability is selected based on fixed probability or number of neighbors regardless of nodes battery capacity whereas in energy aware schemes energy inefficient node could be part of an established path. Therefore, in an attempt to remedy the paucity of research and to address the gaps identified in this area, a study examining the impact of residual energy on forwarding probability and how to exclude small amount of residual battery level during route establishment are critically important. Therefore, our next research plan is to examine the impact of residual energy on forwarding probability and how to exclude small amount of residual battery level during route establishment.

\section{REFERENCES}

[1] Charles E. Perkins, “Ad Hoc Networking”, Addison-Wesley Professional, 2001.

[2] C. Siva Ram Murthy and B.S. Manoj, "Ad Hoc Wireless Networks: Architectures and Protocols, Portable Documents", Pearson Education, 2004.

[3] S. Pleisch, M. Balakrishnan, K. Birman and R.V. Renesse, "MISTRAL: Efficient Flooding in Mobile Ad-hoc Networks," Proceedings of $7^{\text {th }}$ ACM International Symposium on Mobile Ad Hoc Networking and Computing, pp. 1-12, 2006.

[4] Yu-Chee Tseng, Sze-Yao Ni, Yuh-Shyan Chen and JangPing Sheu, "The Broadcast Storm Problem in a Mobile Ad Hoc Network", Wireless Networks, Vol. 8, No. 2, pp. 153167, 2002.

[5] Ya Xu, John Heidemann and Deborah Estrin, "GeographyInformed Energy Conservation for Ad Hoc Routing”, 
Proceedings of $7^{\text {th }}$ Annual International Conference on Mobile Computing and Networking, pp. 70-84, 2001

[6] R. Oliveira, L. Bernardo and P. Pinto, "Flooding Techniques for Resource Discovery on High Mobility MANETs", Available http://www.ctr.kcl.ac.uk/IWWAN2005/papers/41.pdf

[7] Y. Sasson, D. Cavin and A. Schiper, "Probabilistic Broadcast for Flooding in Wireless Mobile Ad Hoc Networks", Proceedings of IEEE Wireless Communications and Networking conference, pp. 1-8, 2003.

[8] D.B. West, "Introduction to Graph Theory", $2^{\text {nd }}$ Edition, Prentice Hall, 2001.

[9] Dietrich Stauffer and Ammon Aharony, "Introduction to Percolation Theory", $2^{\text {nd }}$ Edition, Taylor and Francis, 1994.

[10] Y.C. Tseng, S.Y. Ni, Y.S. Chen and J.P. Sheu, "The Broadcast Storm Problem in a Mobile Ad Hoc Network", Wireless Networks, Vol. 8, No. 2-3, pp. 153-167, 2002.

[11] Jie Wu and Wei Lou, "Forward-Node-Set-based Broadcast in Clustered Mobile Ad Hoc Networks", Wireless Communications and Mobile Computing, Vol. 3, No. 2, pp. 155-173, 2003.

[12] W. Peng and X.C. Lu, "On the Reduction of Broadcast Redundancy in Mobile Ad Hoc Networks", Proceedings of ACM Symposium on Mobile and Ad Hoc Networking and Computing, pp. 129-130, 2000.

[13] M. Bani-Yassein, M. Ould-Khaoua, L.M. Mackenzie and S. Papanastasiou, "Performance Analysis of Adjusted Probabilistic Broadcasting in Mobile Ad Hoc Networks", International Journal of Wireless Information Networks, Vol. 13, No. 2, pp. 127-140, 2006.

[14] Z. Qi and D.P. Agrawal, "Dynamic Probabilistic Broadcasting in Mobile Ad hoc Networks", Proceedings of IEEE 58 ${ }^{\text {th }}$ Vehicular Technology Conference, pp. 1-6, 2003.

[15] Venetis Kanakaris, David Ndzi and Kyriakos Ovaliadis, "Improving AODV Performance using Dynamic Density Driven Route Request Forwarding”, International Journal of Wireless and Mobile Networks, Vol. 3, No. 3, pp.16-27, 2011.

[16] B. Sundaravadivazhagan and P. Jaganathan, "Adaptive Threshold Probabilistic Counter based Broadcast Scheme for Mobile Ad Hoc Networks n Route Discovery", Asian Journal of Information Technology, Vol. 13, No. 9, pp. 569574, 2014.

[17] S.O. Al-Humoud, L.M. Mackenzie, M. Ould-Khaoua and J.M. Abdulai, "RAD Analysis of Adjusted Counter-Based Broadcast in MANETs", Proceedings of $24^{\text {th }} U K$ Performance Engineering Workshop, pp. 1-6, 2008.

[18] Sarah Omar al-Humoud, Lewis M. Mackenzieand Jamaldeen Abdulai, "Neighbourhood-Aware Counter-based Broadcast Scheme for Wireless Ad Hoc Networks", IEEE Global Communication Workshops, pp. 1-6, 2008

[19] Y.C. Tseng, S.Y. Ni and E.Y. Hih, "Adaptive Approaches to Relieving Broadcast Storms in a Wireless Multi-hop Mobile Ad-hoc network", IEEE Transactions on Computers, Vol. 52, No. 5, pp. 545-557, 2003.

[20] Y.B. Ko and N.H. Vaidya, "Location-Aided Routing (LAR) in Mobile Ad Hoc Networks", Proceedings of $4^{\text {th }}$ Annual ACM/IEEE International Conference on Mobile Computing and Networking, pp. 1-4, 1998.
[21] Sandra M. Hedetniemi, Stephen T. Hedetniemi and Arthur L. Liestman, "A Survey of Gossiping and Broadcasting in Communication Networks", Networks, Vol. 18, No. 4, pp. 319-349, 1988.

[22] Z. J. Haas, J. Y. Halper and L. Li, "Gossip Based Ad Hoc Routing”, IEEE Transactions on Networking, Vol. 14, No. 3, pp. 479-491, 2006.

[23] Pradeep Kyasanur, Romit Roy Choudhury and Indranil Gupta, "Smart Gossip: An Adaptive Gossip-based Broadcasting Service for Sensor Networks", Proceedings of IEEE International Conference on Mobile Adhoc and Sensor Systems, pp. 91-100, 2006.

[24] B. Chen, K. Jamieson, R. Morris and H. Balakrisnan, "Span: An Energy-Efficient Coordination Algorithm for Topology Maintenance in Ad Hoc Wireless Networks", Wireless Networks, Vol. 8, No. 5, pp. 481-494, 2002.

[25] A.V. Kini, V. Veeraraghavan, N. Singhal and S. Weber, "SmartGossip: An Improved Randomized Broadcast Protocol for Sensor Networks", Proceedings of $5^{\text {th }}$ International Conference on Information Processing in Sensor Networks, pp. 210-217, 2006.

[26] S. Singh, M. Woo and C. Raghavendra, "Power Aware Routing in Mobile Ad Hoc Network", Proceedings of International Conference on Mobile Computing and Networking, pp. 181-190, 1998.

[27] A. Srinivas and E. Modiano, "Minimum Energy Disjoint Path Routing in Wireless Ad-Hoc Networks", Proceedings of $9^{\text {th }}$ Annual International Conference on Mobile Computing and Networking, pp. 122-133, 2003.

[28] M. W. Subbarao, "Dynamic Power-Conscious Routing for MANET: An Initial Approach", Proceedings of 50 $0^{\text {th }}$ IEEE Vehicular Technology Conference, Vol. 2, pp. 1232-1237, 1999.

[29] J. Gomez and A. Campbell, "Power-Aware Routing Optimization for Wireless Ad Hoc Networks", Proceedings of High Speed Networks Workshop, pp. 1-6, 2001.

[30] J. Gomez, A.T. Campbell, M. Naghshineh and C. Bisdikian. "Conserving Transmission Power in Wireless Ad Hoc Networks", Proceedings of $9^{\text {th }}$ International Conference on Networking Protocols, pp. 1-6, 2001.

[31] K. Scott and N. Bamboos, "Routing and Channel Assignment for Low Power Transmission in PCS", Proceedings of $5^{\text {th }}$ IEEE International Conference on Universal Personal Communications, pp. 498-504, 1996.

[32] C.S. Raghavendra and Suresh Singh, "PAMAS-Power Aware Multi-Access Protocol with Signaling for Ad Hoc Networks", ACM Communications Review, Vol. 28, No. 3, pp. 1-20, 1998.

[33] S. Doshi, S. Bhandare and T.X Brown, "An On-Demand Minimum Energy Routing Protocol for a Wireless Ad Hoc Network", ACM Mobile Computing and Communications Review, Vol. 6, No. 3, pp. 50-66, 2002.

[34] V. Rodoplu and T. Meng, "Minimum Energy Mobile Wireless Networks", IEEE Journal on Selected Areas on Communications, Vol. 17, No. 8, pp. 1333-1344, 1999.

[35] S. Banerjee and A. Misra, "Minimum Energy Paths for Reliable Communication in Multi-hop Wireless Networks", Proceedings of $3^{\text {rd }}$ ACM International Symposium on Mobile Ad Hoc Networking and Computing, pp. 146-156, 2002. 
[36] Jinhua Zhu, Chunming Qiao and Xin Wang, "A Comprehensive Minimum Energy Routing Protocol for Wireless Ad Hoc Networks", Proceedings of $23^{\text {rd }}$ Annual Joint Conference of the IEEE Computer and Communications Societies, pp. 121-125, 2004.

[37] C.K. Toh, "Maximum Battery Life Routing to Support Ubiquitous Mobile Computing in Wireless Ad Hoc Networks", IEEE Communications Magazine, Vol. 39, No. 6, pp. 138-147, 2001.

[38] Jin-Man Kim and Jong-Wook Jang, "AODV based ENERGY Efficient Routing Protocol for Maximum Lifetime in MANET", Proceedings of IEEE Advanced International Conference on Telecommunications, Internet and Web Applications and Services, pp. 27-32, 2006.

[39] X. Wang, L. Li and C. Ran, "An Energy-Aware Probability Routing in MANETs", Proceedings of IEEE Workshop on IP Operations and Management, pp. 146-151, 2004.

[40] Kyungtae Woo, Chansu Yu, Dongman Lee, Hee Yong Youn and B. Lee, "Non-Blocking, Localized Routing Algorithm for Balanced Energy Consumption in Mobile Ad Hoc Networks", Proceedings of $9^{\text {th }}$ International Symposium on Modeling, Analysis and Simulation of Computer and Telecommunication Systems, pp. 117-124, 2001.

[41] Malek Al-Gabri et.al., "Improved the Energy of Ad hoc on Demand Distance Vector Routing Protocol", Proceedings of International Conference on Future Computer Supported Education, Vol. 2, pp. 355-361, 2012.

[42] Wang Kun, Xu Yin-Long, Chen Guo-Liang and Wu YaFeng, "Power-Aware on-Demand Routing Protocol for MANET", Proceedings of $24^{\text {th }}$ International Conference on Distributed Computing Systems Workshops, pp. 723-728, 2004.

[43] Tai Hieng Tie, Chong Eng Tan and Sei Ping Lau, "Alternate Link Maximum Energy Level Ad Hoc Distance Vector Scheme for Energy Efficient Ad Hoc Networks Routing", Proceedings of IEEE International Conference on Computer and Communication Engineering, pp. 176-182, 2010.

[44] Z. Zhaoxiao, P. Tingrui,and Z. Wenli, "Modified EnergyAware AODV Routing for Ad hoc Networks", Proceedings of IEEE Global Congress on Intelligent Systems, pp. 338342, 2009.

[45] T. Jie, W. Yu, and L. Jianxing, "Researching on AODV and PS-AODV Routing Protocols of Ad Hoc Network for Streaming Media", Proceedings of $2^{\text {nd }}$ International
Conference on Computer Application and System Modeling, pp. 1-4, 2012.

[46] Q. Lei and W. Xiaoqing, "Improved Energy-Aware AODV Routing Protocol", Proceedings of IEEE International Conference on Information Engineering, pp. 18-21, 2009.

[47] Annapurna P. Patil, K. Rajani Kanth, B. Sharanya, M.P. Dinesh Kumar and J. Malavika, "Design of an Energy Efficient Routing Protocol for MANETs based on AODV", International Journal of Computer Science Issues, Vol. 8, No 1, pp. 215-220, 2011.

[48] R. Vinod Kumar and R.S.D. Wahida Banu, "E²AODV: Protocol for Load Balancing in Ad-Hoc Networks", Journal of Computer Science, Vol. 8, No. 7, pp. 1198-1204, 2012.

[49] Ashwini V. Biradar, Shrikant R. Tandle and Veeresh G. Kasabegoudar, "Detailed Performance Analysis of Energy based AODV Protocol in Comparison with Conventional AODV, and DSDV Protocols in MANET", International Journal of Computer Applications, Vol. 49, No. 10, pp. 49 $58,2012$.

[50] N. Gupta and S.R. Das, "Energy-Aware On-Demand Routing for Mobile Ad Hoc Networks", Proceedings of the $4^{\text {th }}$ International Workshop on Distributed Computing, Mobile and Wireless Computing, pp. 164-173, 2002.

[51] Sunil Tanejaa, Ashwani Kush, Amandeep Makkar and Bharat Bhushan," "Power Management in Mobile Ad hoc Network", International Transaction Journal of Engineering, Management, and Applied Sciences and Technologies, Vol. 2, No. 2, pp. 215-225, 2011.

[52] J. Zhu and X. Wang, "Peer: A Progressive Energy Efficient Routing Protocol for Wireless Ad Hoc Networks", Proceedings of $24^{\text {th }}$ Annual Joint Conference of the IEEE Computer and Communications Societies, Vol. 3, pp. 18871896, 2005.

[53] Young-Bae Ko and Nitin H. Vaidya, "Location-Aided Routing (LAR) in Mobile Ad Hoc Networks", Wireless Networks, Vol. 6, No. 4, pp. 307-321, 1998.

[54] I. Stojmenovic, and X. Lin, "Power-Aware Localized Routing in Wireless Networks", IEEE Transactions on Parallel and Distributed Systems, Vol. 12, No. 11, pp. 1122 1133,2001

[55] G. Girling, J. Wa, P. Osborn and R. Stefanova, "The Design and Implementation of a Low Power Ad Hoc Protocol Stack", Proceedings of IEEE Wireless Communications and Networking Conference, pp. 225-229, 2000. 